\title{
THE PROPERTIES OF PLANETARY NEBULAE NUCLEI: STELLAR WINDS
}

\author{
L. BIANCHI and G. DE FRANCESCO
}

Osservatorio Astronomico di Torino, Strada Osservatorio, 20, 10025 Pino Torinese, Torino, Italy

We present IUE observations of some nuclei of Planetary Nebulae. From these data we derive the stellar photospheric parameters $\left(\mathrm{T}_{e f f}, \mathrm{~L}_{b o l}, \log \mathrm{g}\right)$, and the wind characteristics (velocity, mass loss rate). $\mathrm{T}_{e f f}, \mathrm{R}_{*}, \mathrm{~L}_{b o l}$ are derived from UV low resolution spectra, combining optical and radio data, from Bianchi (1988) or from new IUE data, with the same method (fit of the UV continuum with model atmospheres for high gravity stars, after correcting for reddening and for the contribution of continuum emission by the nebular gas). P Cygni profiles from IUE high resolution spectra are fitted with the SEI method and $V_{x}$ is derived. The non-LTE ionisation in the wind and the mass loss rate are computed as in Bianchi et al. (1986). Details are given in a forthcoming paper. The results for a first group of objects are given in the Table below.

References

Bianchi, L., Cerrato, S., Grewing, M., 1986, Astron. Astrophys. 169, 227.

Bianchi, L., 1988, ESA SP-281, 2, 173.

Bianchi, L., Cascio, F., 1989, in "From Miras to Planetary Nebulae: Which Path for Stellar Evolution", eds. Mennessier and Omont, Editions Frontieres, p. 403.

Bianchi, L., 1991, Astron. Astrophys. 253, 447.

Bianchi, L., De Francesco, G., 1992, in preparation.

Heap, S., R., Augensen, H., J., 1987, Ap. J. 313, 268.

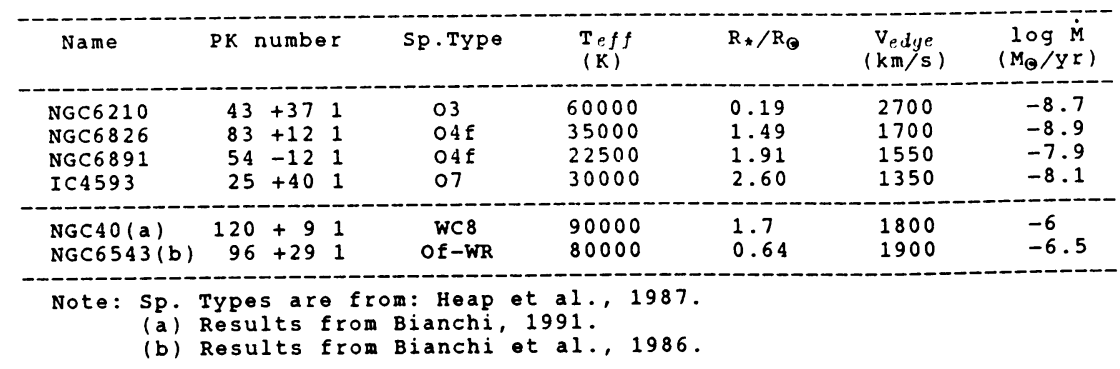

